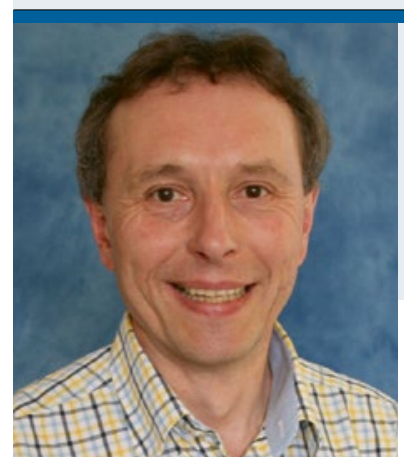

„Und nun das: Angeblich reduzieren Daumenlutschen und Nägelkauen das Sensibilisierungsrisiko gegenüber zahlreichen Umweltallergenen."

Dr. Thomas Hoppen, Chefredakteur

Normales neurokognitives Outcome

\title{
Kindernarkosen machen nicht dumm
}

— Die Sorge um Spätfolgen von Narkosen scheint unbegründet. In einer GeschwisterVergleichsstudie wurden Kinder, die eine Narkose innerhalb der ersten 36 Lebensmonate erhalten hatten, 8-15 Jahre nach dem Eingriff nachuntersucht. Es fanden sich keine Unterschiede bezüglich Neurokognition und Verhalten im Vergleich zu ihren Geschwistern, die nicht narkotisiert worden waren [Sun LS et al. JAMA 2016;315:2312-20].
In die Studie aufgenommen wurden 105 Geschwisterpaare, eines der Geschwister hatte eine Leistenhernienoperation mit einer mittleren Narkosedauer von 80 min erhalten. Eine Botschaft, die wir Kinderärzte anhand dieser Ergebnisse gerne vermitteln werden: Nach einem einmaligen elektiven Eingriff werden Gedächtnis, Lernverhalten, Ausdruck, Sprache, Aufmerksamkeit und Verhalten langfristig nicht beeinträchtigt.

\section{Vorübergehendes Phänomen Blind durch Smartphones?}

— Der Siegeszug der mobilen Medien ist beeindruckend und erdrückend zugleich. Nun wurde über eine mehrminütige intermittierende monookuläre Blindheit bei zwei Patienten infolge einer erhöhten Lichtstärke des Displays im Dunkeln berichtet [AlimMarvasti A et al. N Engl J Med 2016; 374:25024]. Letztendlich handelte es sich nur um eine Folge einseitiger Lichtadaptation. Interessanterweise war immer das Auge betroffen, das sich kontralateral zur Liegeseite im Bett befand. Dieses schauende Auge war also lichtadaptiert, während das durch das Kopfkissen blockierte untere Auge dunkeladaptiert war. Ein physiologisches Phänomen ohne eigentlichen Krankheitswert. Trotzdem warnen Kinderärzte vor dem besorgniserregenden Anstieg von Überforderung, Kopfschmerzen und ADHS bei jungen Menschen und sie sehen einen naheliegenden Zusammenhang mit der wachsenden Nutzung digitaler Medien bereits im Kindergartenalter.

\section{Tiergestützte Therapie Elefanten helfen bei Down-Syndrom}

— Andere Länder, andere

Therapien. In einem Forschungsartikel aus Thailand wird der Einfluss eines Förderprogramms für Kinder mit Trisomie 21 mit etwas ungewöhnlichen Co-Therapeuten vorgestellt [Satiansukpong $\mathrm{N}$ et al. Occup Ther Int 2016;23:121-31]. Der Einfluss von Elefanten wurde an 16 Patienten verglichen. Die eine Gruppe erhielt lediglich regulären Schulunterricht, die zweite Gruppe eine spezielle zusätzliche Therapie mit Elefantenassistenz zweimal pro Woche über 2 Monate. Es fand sich ein positiver Einfluss auf die Grob- und Feinmotorik der behandelten Kinder. Elefanten zeichneten sich als effektive Motivationsfaktoren aus. Also ein Argument mehr für den Einsatz von tierassistierten Therapieverfahren zur Verbesserung der körperlichen, sozialen, emotionalen und kognitiven Entwicklung.

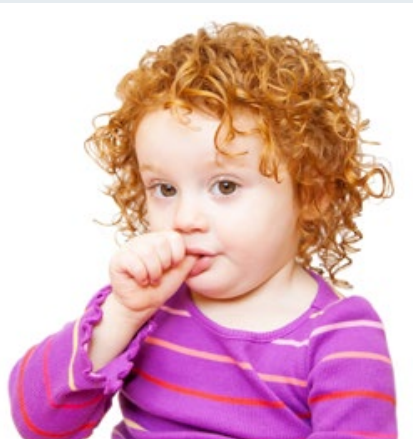

Futter für die Hygienehypothese Daumenlutschen schützt vor Atopie

— Viele Eltern setzen alles daran, bei ihren Kindern das Lutschen an den Fingern oder Kauen an den Nägeln zu unterbinden. Wirksame Interventionsprogramme dazu wurden entwickelt. Und nun das: Angeblich reduzieren beide Verhaltensweisen das Sensibilisierungsrisiko gegenüber zahlreichen Umweltallergenen. Analysiert wurde eine Gruppe von rund 1.000 Menschen über einen Zeitraum von 38 Jahren, die 1972/73 in der neuseeländischen Küstenstadt Dunedin geboren worden waren [Lynch SJ et al. Pediatrics 2016;138: pii: e20160443]. Dabei fanden mehrere Elternbefragungen zum Daumenlutschen- und Nägelkauen-Verhalten der Kinder und standardisierte HautPrick-Testungen im Alter von 13 und 32 Jahren statt. Kinder, die im Alter von 5-11 Jahren am Daumen lutschten oder an den Nägeln kauten, hatten mit $13(p=0,013)$ und $32(p=0,001)$ Jahren ein geringeres Risiko für eine atopische Sensibilisierung verglichen mit Nichtlutschern bzw. Nichtkauern - am besten schnitten die Kinder ab, die sogar beidem frönten. Die zugleich beobachtete fehlende Assoziation zu Heuschnupfen oder Asthma bleibt zu diskutieren. Die Kernerkenntnis unterstützt jedoch die Hygienehypothese, dass die Aufnahme von Mikroben das Darmmikrobiom beeinflusst und eine präventive immunologische Reaktion zur Folge hat. Oder andersherum: Wird die Exposition zur oralen Aufnahme von Umweltmikroben in jungen Jahren eingeschränkt, steigt das Risiko einer Sensibilisierung gegenüber inhalativen Allergenen im Laufe des Lebens stärker an. 